Physica B 171 (1991) 10-19

North-Holland

\title{
Local and band approaches to actinide systems
}

\author{
J.W. Rasul \\ Physics Department, University of Michigan, Ann Arbor, MI 48109, USA \\ Invited paper.
}

\begin{abstract}
We investigate two models of interest in the context of actinide and rare earth systems. We extend previous work on the Anderson model for two magnetic configurations and obtain the singlet quasiparticle energy to next leading order in the $1 / N$ expansion. We prove that the appropriate Ward identity and perturbative scaling results are reproduced in this theory. We also prove the opposite limit in which there is significant $\mathrm{f}-\mathrm{f}$ overlap using the infinite $U$ Hubbard model. Working to second order in the $1 / N$ expansion, we study the quasiparticle scattering amplitudes and find a large enhancement in the Landau parameter $f_{0}^{a}$ near half filling which varies as the cube of the mass enhancement. For a free-electron-like dispersion relation, this implies a transition to a ferromagnetic state for small hole concentrations.
\end{abstract}

\section{Introduction}

The rapid growth in the study of correlated electron systems continues to leave as many unanswered questions as it poses new ones. The high- $T_{\mathrm{c}}$ problem has focussed most theoretical attention on the properties of doped Mott insulators [1], although the heavy-fermion perspective of keeping separate bands is another promising line of inquiry [2]. More recent work looks to a completely different paradigm, namely that of interacting one-dimensional Fermi systems as providing the appropriate starting point for discussing the normal state [3].

Although the heavy-fermion systems may provide an analogy by which the high- $T_{\mathrm{c}}$ problem may be better appreciated, they still require better understanding in themselves. Under this label falls a large range of cerium, ytterbium and other rare earth systems as well as the actinides and their alloys and compounds. While the field has seen tremendous progress as regards the understanding of dilute cerium and ytterbium systems, the lattice problem, as well as the extensions to other dilute rare earth and actinide systems, still remains unclear.

The lattice problem will perhaps require much newer insight before it can be claimed to be understood. The same may be true for dilute actinide and rare earth systems such as those involving, e.g., thulium impurities. In the meantime, it is reasonable to push those techniques that have worked for cerium as far as possible, if only to understand their limitations. In the next section, we will review work carried out over the last few years on this problem and present an extension of the $1 / N$ technique [4], that has worked so well for cerium, to this question. The extension concerns the corrections at next leading order to the quasiparticle energy of the renormalized singlet state which emerges in leading order as the ground state. We will show that the corrections satisfy both the low energy Ward identity results, namely the connection between Wilson ratio and charge susceptibility [5], as well as the high energy properties obtained from perturbative scaling [6].

The actinide lattice systems asre also interesting from another point of view. Those that have appreciable $f-f$ overlap may still maintain the large degree of correlation associated with high angular momentum states. In other words, $U$ for the $f$ shells may be so large that the Hubbard model for these actinide systems lies outside the regime for which the conventional Stoner-LDA approaches are valid. 
The Hubbard model for these systems may well lie in the parameter range for which a $t-J$ model is an appropriate effective Hamiltonian. Going further, we may regard the antiferromagnetic term as unimportant if the filling is sufficiently large. We then have a model of electrons moving in a lattice in which double occupancy is forbidden. This then reduces to the infinite $U$ Hubbard model which has itself received a fair amount of attention in connection with the high- $T_{\mathrm{c}}$ problem [7]. The difference is that we are interested in three dimensions rather than in two dimensions, and the charge-spin separation aspects particular to two dimensions are no longer an issue. It is known that for a single hole in an infinite $U$ Hubbard model Nagaoka's theorem [8] proves that the system gains the most kinetic energy if the matrix elements for hole propagation are largest and uniform. This requires that the system be a ferromagnet.

Starting from the opposite limit of large hole concentration, we expect a metal with a moderate mass enhancement, because of the restricted hopping nature of the infinite $U$ constraint. As the electron filling is increased, the mass enhancement increases as there are fewer empty atoms to hop onto and it is natural to ask what the interactions are between these quasiparticles. Experience with the Anderson lattice suggests a natural way of proceeding along these lines, namely the $1 / N$ expansion [9]. For the infinite $U$ Anderson lattice model, the leading order yields a hybridized band structure, renormalized by the effects of the infinite $U$ constraint. The large $N$ limit picks out the degenerate Fermi liquid ground state over other magnetically ordered ones, and the remaining self-consistency condition to be satisfied is merely the Luttinger theorem requirement, namely the number of states enclosed by the Fermi surface is the same as in the noninteracting system.

The quasiparticle interactions can then be analyzed following standard loop expansion methods. The problem with the $1 / N$ expansion is that the one loop results only reflect the effects of a single density fluctuation exchange. The reason that only density fluctuations appear at this order lies in the way the $1 / N$ expansion is formulated - the Bose operators that restrict the hopping to empty sites are coupled to the total $f$ electron density at each site. To obtain spin fluctuation effects, one has to work to higher orders which becomes rapidly more difficult. However, if one wishes to work in a controlled fashion, an expansion parameter of some sort is necessary, and $1 / N$ is so far the only known expansion parameter that gives nondivergent results for infinitely strongly correlated systems.

Anderson lattice calcualtions to $1 / N^{2}$ order were carried out by Houghton, Read and Won [10], who obtained a substantial correction to quasiparticle scattering amplitudes from the RKKY interaction. In the second part of this paper, we shall perform a similar calculation for the infinite $U$ Hubbard model. In the Anderson lattice calculation, Houghton et al. obtained the RKKY interaction as a result of virtual processes between the conduction electrons and the high frequency charge fluctuation part of the f electron spectral density. These high energy intermediate processes do not get screened in the Kondo fashion, hence the appearance of the RKKY energy.

In the case of interest to us, namely the infinite $U$ Hubbard model, there is no RKKY interaction for us to search for, but there is a high frequency coherent part in the charge fluctuation spectrum, namely the zero sound pole. We shall show that interactions between the renormalized quasiparticles and this collective mode lead to substantial corrections to the scattering amplitudes. In the Cooper pair channel these prove to be repulsive, indicating strong ferromagnetic spin fluctuations. We are thus able to make contact with the philosophy of the Stoner theory, except that we are translating a critical concentration of carriers into a critical exchange interaction defining the Stoner criterion.

\section{Low temperature properties of the two magnetic state Anderson model}

To describe rare earth systems other than $\mathrm{Ce}$ or $\mathrm{Yb}$ as well as actinide systems realistically, it is inevitable that at some point the Anderson model fluctuating between two degenerate configurations 
has to be addressed. Early work on this problem treated impurity spin states of either spin $\frac{1}{2}$ or spin 1 coupled to band electrons of spin $\frac{1}{2}$ [11]. These models and their generalizations have been solved exactly and their ground states are found to be magnetic [12]. Another possibility is that the crystal fields conspire to make the lowest atomic configuration nonmagnetic. The dominant interactions are then quadrupolar charge fluctuations [13].

If we wish to allow band and local states of the same symmetry to couple, then we must allow for the case where they have the same number of internal degrees of freedom. In this case we expect the impurity spin to be completely compensated, as in the Kondo problem. The first calculations to show this were variational, by Yafet et al. [14], who obtained singlet ground states with very small binding energies throughout the range of valence. A $1 / N$ scheme that gave the same results at zero temperature was developed to leading order by Read et al. [15]. The same low energy scale was found in a variational study of the $\mathrm{f}^{2}-\mathrm{f}^{3}$ Anderson mode by Nunes et al. [16]. Other bulk properties have been discussed within this scheme [17].

Extensions along the lines of the noncrossing approximation have also been developed, and so far actual calculations have only been performed at a level equivalent to keeping the leading $1 / N$ processes [18]. The numerical RG method has now been applied to the simplest $f^{1}-f^{2}$ problem and the physical conclusion of this work is that the ground state is indeed a compensated singlet [19].

In order to develop conventional many-body techniques that have a wide range of application and are more readily understood, it seems reasonable, therefore, to extend the low order approaches, confident that they have at least the essential physics correct. In this paper, we shall work within the $1 / N$ framework since it allows us to keep track of different processes and control our calculation. While the starting model is not particular realistic, consisting as it does of spin $j$ electrons interacting with impurity states labeled by the same indices, but with either one or two electrons on the site, once the correct graphs have been selected, it is straightforward to adapt the model to more realistic coupling schemes.

To attack the problem of an Anderson model fluctuating between two magnetic configurations, we could adopt one of a number of $1 / N$ approaches. If we are to discuss low temperature properties we would rather avoid the nonanalyticities that plague self-consistent approaches at low energies and a direct resummation of the perturbation series in powers of the mixing is perfectly adequate [4]. The key is to incorporate the $\mathrm{f}^{(0}$ singlet state into the initial Hamiltonian if we want to obtain a ground state with the correct symmetry. The Hamiltonian is then

$$
\begin{aligned}
H= & \sum_{k, m=-j}^{j} \epsilon_{k} c_{k m}^{+} c_{k m}+V \sum_{k, m}\left\{|1 m\rangle\left\langle 2 m m^{\prime}\left|c_{k m}^{+}+c_{k m^{\prime}}\right| 2 m m^{\prime}\right\rangle\langle 1 m|\right\} \\
& +E_{1} \sum_{m}|1 m\rangle\left\langle 1 m\left|+E_{2} \sum_{m, m^{\prime}}\right| 2 m m^{\prime}\right\rangle\left\langle 2 m m^{\prime}\right|+V_{0} \sum_{k, m}\left\{c_{k m}^{+}|0\rangle\langle 1 m|+| 1 m\rangle\langle 0| c_{k m}\right\},
\end{aligned}
$$

where $\epsilon_{k m}$ denotes the band energy in spin state $m, V$ denotes the $\mathrm{f}^{1}-\mathrm{f}^{2}$ mixing element and $E_{1}$ and $E_{2}$ are the bare energies of the $\mathrm{f}^{1}$ and $\mathrm{f}^{2}$ configurations. The extra mixing term into the $\mathrm{f}^{\prime}$ state allows the use of the 'quasiparticle energy' scheme developed by Keiter and Kimball [20] for the $f^{0}-f^{1}$ problem. While the interpretation of this scheme should be carried out with care, it does at least yield correct results at low temperatures and saves a considerable amount of effort over other perturbative resummation schemes for the $\mathrm{f}^{1}-\mathrm{f}^{2}$ problem. The leading order results obtained by Read et al. are in terms of a vertex function satisfying an integral equation (see fig. 1) 


$$
\int_{-\infty}^{\infty} \mathrm{d} \epsilon^{\prime} k\left(\epsilon, \epsilon^{\prime}\right) \beta\left(\epsilon^{\prime}\right)=0
$$

where the integral operator is given by

$$
k\left(\epsilon, \epsilon^{\prime}\right)=\alpha(\epsilon) \delta\left(\epsilon-\epsilon^{\prime}\right)-\frac{N V^{2} f\left(\epsilon^{\prime}\right)}{L\left(\epsilon, \epsilon^{\prime}\right)}
$$

where

$$
\alpha(\epsilon)=E_{1}-E-\epsilon-N V^{2} \sum_{\epsilon^{\prime}} \frac{f\left(\epsilon^{\prime}\right)}{L\left(\epsilon, \epsilon^{\prime}\right)},
$$

and $L\left(\epsilon, \epsilon^{\prime}\right)=E_{2}-E-\epsilon-\epsilon^{\prime}$. The presence of an integral operator here shows the difficulty of the $f^{1}-f^{2}$ problem compared with the cerium case. In the limit of zero temperature, eq. (2) agrees with the results of variational approaches [14]. Higher order corrections are obtained by dressing the $\mathrm{f}^{1}$ and $\mathrm{f}^{2}$ intervals with processes involving a single particle-hole pair. This leads to the appearance of other vertex parts such as that shown in fig. 2, which satisfy integral equations involving the above kernel. One way in which these vertex functions contribute to the quasiparticle energy is shown in fig. 3. By swapping the order in which the hole lines with energies $\epsilon_{1}$ and $\epsilon_{2}$ emerge at the left hand side, we obtain another set of contributions. All these processes are simply interpreted as dressing either the $\mathrm{f}^{1}$ or $\mathrm{f}^{2}$ lines, and the integral eq. (2) may be corrected to read

$$
\int_{-\infty}^{\infty} k\left(\epsilon, \epsilon^{\prime}\right) \beta\left(\epsilon^{\prime}\right) \mathrm{d} \epsilon^{\prime}=V^{4} N \int \frac{\mathrm{d} \epsilon_{2} \mathrm{~d} \epsilon^{\prime} f\left(\epsilon_{2}\right)\left[1-f\left(\epsilon^{\prime}\right)\right]}{L\left(\epsilon_{1}+\epsilon_{2}\right)}\left[Q\left(\epsilon_{1}, \epsilon_{2} ; \epsilon^{\prime}\right)+Q\left(\epsilon_{2}, \epsilon_{1} ; \epsilon^{\prime}\right)\right],
$$

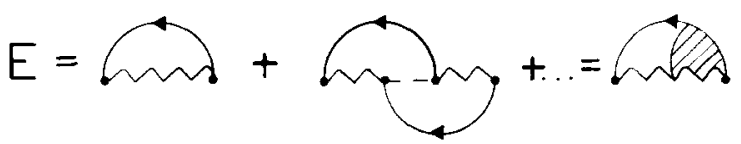

(a)

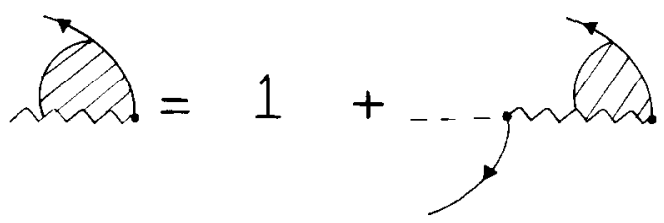

(b)

Fig. 1. Contributions to (a) the quasiparticle scattering energy $E$ involving the vertex function (b).

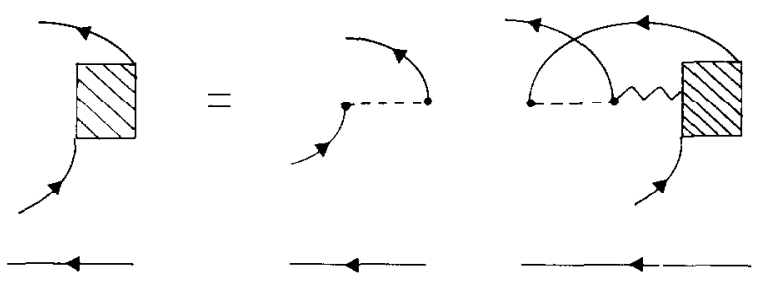

Fig. 2. A vertex function entering the next order corrections. 


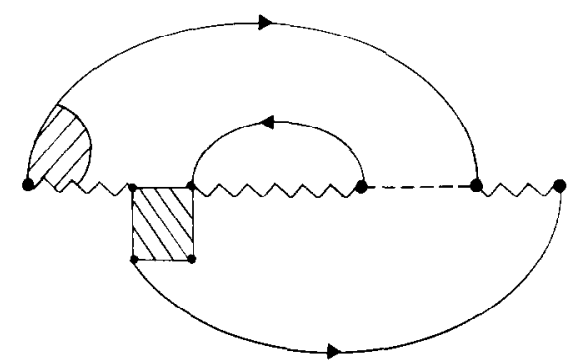

Fig. 3. A contribution to the singlet quasiparticle energy $E$ involving the higher order vertex corrections.

where $Q\left(\epsilon_{1}, \epsilon_{2} ; \epsilon^{\prime}\right)$ satisfies

$$
\int_{-\infty}^{\infty} k\left(\epsilon_{1}+\epsilon_{2}-\epsilon^{\prime}, \epsilon^{\prime \prime}\right) Q\left(\epsilon_{1}, \epsilon^{\prime \prime} ; \epsilon^{\prime}\right)=\frac{B\left(\epsilon_{1}\right)+\beta\left(\epsilon_{2}\right)}{L\left(\epsilon_{1}+\epsilon_{2}\right)} .
$$

These integral equations determine the single quasiparticle energy correct to order $1 / N$. To ensure that the low energy properties are being properly described, we should check that the Wilson ratio (susceptibility/linear specific heat) is given correctly. At leading order, the magnetic field and temperature affect the singlet energy in much the same way since they both enter via the Fermi function for band holes. The spectrum is that of electrons, so the Wilson ratio reduces to unity in this case. The $1 / N$ corrections, however, bring in particle-hole pairs with the same spin and these have a bosonic character at low energies. They, therefore, do not couple to the magnetic field and cannot affect the susceptibility but they do contribute to the specific heat, therefore, the Wilson ratio is changed from its zero order value of unity. Extracting this Bose-like part requires writing the particle-hole combination of Fermi functions as follows,

$$
f(\epsilon, m)\left[1-f\left(\epsilon^{\prime}, m\right)\right]=\left\{f\left(\epsilon^{\prime}, m\right)-f(\epsilon, m)\right\} b\left(\epsilon-\epsilon^{\prime}\right)
$$

where $b(\epsilon)$ is the Bose function. Expanding this Bose function at low temperatures yields the extra contributions to the specific heat mentioned above. Care has to be taken in extracting the low energy behavior of the vertex function as it is in fact singular at low particle-hole energies,

$$
Q\left(\epsilon_{1}, \epsilon_{2}, \epsilon^{\prime}\right)=\frac{-B\left(\epsilon_{2}\right) q\left(\epsilon_{1}\right)}{\left(\epsilon_{1}-\epsilon^{\prime}\right)}+\text { reg. part }
$$

where

$$
q(\epsilon)=\int_{-\infty}^{\infty} k\left(\epsilon, \epsilon^{\prime}\right) \beta\left(\epsilon^{\prime}\right)^{2} \mathrm{~d} \epsilon^{\prime} .
$$

This emphasizes the importance of working rigorously within the $1 / N$ procedure. Arbitrary resumma- 
tion procedures can easily yield divergent results. After some manipulation, the Wilson ratio takes the correct form,

$$
R=\chi / \gamma=1+\frac{1}{N}-\frac{\chi_{\mathrm{ch}}}{N \gamma}
$$

where $\chi_{\mathrm{ch}}$ is the charge susceptibility $\mathrm{d}^{2} E / \mathrm{d} E_{12}^{2}$. This agrecs with the Ward identity result of Yoshimori [5] for the finite $U$ Anderson model generalized to the degenerate case by Schlottmann [5]. The result essentially counts the number of channels present in the low energy spectrum. One is due to the charge fluctuations, while the remaining $N-1$ arise from the spin degrees of freedom.

The high energy region is also interesting to examine as the charge fluctuation energy scales obtained by perturbative scaling should emerge from the theory. Into these scales is absorbed all the dependence of the theory on bare quantities such as the ionic energy difference and the electron bandwidth. From eq. (6), it is simple to show that, for large energies,

$$
Q\left(\epsilon_{1}, \epsilon_{2} ; \epsilon^{\prime}\right)=\frac{1}{\left(\epsilon^{\prime}-\epsilon_{1}-\epsilon_{2}\right)} \frac{\left[\beta\left(\epsilon_{1}\right)+\beta\left(\epsilon_{2}\right)\right]}{\left(E_{2}-E_{0}-\epsilon_{1}-\epsilon_{2}\right)},
$$

and consequently the second term in eq. (5) may be absorbed into the energy denominator of the right hand side of eq. (3). We may regard our corrections as yielding a shift in both the $\mathrm{f}^{\prime}$ and $\mathrm{f}^{2}$ configurational energies proportional to $\tilde{\Gamma} \ln D$ (where $\tilde{\Gamma}=N \rho_{0} V^{2}$ ). The scale on which charge fluctuations occur is then not the bare ionic energy difference but the scale $T^{*}$, given by

$$
\left|T^{*}\right|=E_{2}-E_{1}+(N-3) \frac{\tilde{\Gamma}}{N} \ln \frac{D}{\left|T^{*}\right|},
$$

which is precisely the scaling invariant obtained earlier by Read et al. [15].

We have, therefore, obtained a theory that is consistent at both high and low energies and should form a basis for calculating other properties such as the coefficients of the low temperature expansion of the specific heat susceptibility which are of central importance for static measurements on heavyfermion systems. Spectral properties should also be calculable to the order to which we have worked here.

\section{Ferromagnetic instability in the infinite $U$ Hubbard model}

The starting point for our discussion of the infinite $U$ Hubbard model is the auxiliary boson version of the Lagrangian;

$$
\begin{aligned}
L(\tau)= & \sum_{i} b_{i}^{+} \frac{\partial}{\partial \tau} b_{i}+\sum_{i m} f_{i m}^{+}\left(\frac{\partial}{\partial \tau}-\mu\right) f_{i m}+\sum_{i j} t_{i j} f_{i m}^{+} f_{j m} b_{i} b_{j}^{+} \\
& +\sum_{i} \mathrm{i} \lambda_{i}\left[\sum_{m} f_{i m}^{+} f_{i m}+b_{i}^{+} b_{i}-Q\right],
\end{aligned}
$$


where the bosons $b_{i}$ label the empty state at sitc $i, f_{i m}^{+}$creates a fermion in spin channel $m$ (which runs from $-j$ to $+j), t_{i j}$ is the transfer matrix element, $\mu$ is the chemical potential and $Q$ is the total charge which is scaled by the degeneracy $N=2 j+1$ in order to obtain a sensible large $N$ limit. The last term in eq. (13) enforces the $U=\infty$ constraint and couples fluctuations in the Bose field to the total charge density. Therefore, the bosons represent density fluctuations only in this model. After performing the standard radial gauge transformation [4], the partition function of this model can be written to Gaussian $(1 / N)$ order as:

$$
Z=Z_{\text {mean ficld }} \exp \{\operatorname{Tr} \ln S(k, \omega)\}
$$

where the matrix elements of the Bose propagator are

$$
\begin{aligned}
& S_{11}(\underline{k}, \omega)=\mathrm{i} \lambda-\frac{N}{\beta} \sum_{\underline{\underline{p}}} t_{\underline{k}+\underline{p}} G(\underline{p})+\frac{N \rho^{2}}{2} I_{2}(\underline{k}, \omega) \text {, } \\
& S_{12}(\underline{k}, \omega)=\rho+\frac{1}{2} N \rho I_{1}(k, \omega) \text {. } \\
& S_{22}(\underline{k}, \omega)=\frac{1}{2} N I_{0}(\underline{k}, \omega) \text {, }
\end{aligned}
$$

where

$$
I_{n}(\underline{k}, \omega)=\frac{1}{\beta} \sum_{p_{i}} G(p) G(p+k)\left[t_{\underline{p}}+t_{\underline{k}+\underline{p}}\right]^{n},
$$

and

$$
G(p)=\left(-\mathrm{i} \omega+E_{p}\right)^{-1} .
$$

To recap, the mean field theory [21] results yield a renormalized band with energies

$$
E_{k}=\rho^{2} t_{k}+\mathrm{i} \lambda
$$

so that the band is narrowed by a factor

$$
\rho^{2}=Q-n_{\mathrm{f}},
$$

where $n_{\mathrm{f}}$ denotes the filling per spin channel. This may naturally be interpreted as a mass enhancement. The remaining factor $i \lambda$ denotes a shift in the chemical potential. Thus the leading order corresponds to a system of noninteracting quasiparticles. To obtain response functions and explore quasiparticle interactions, we need to make use of the Gaussian part of eq. (14). We find that the charge susceptibility takes the form

$$
\chi_{\mathrm{ch}}(\underline{k}, \omega)=\rho^{2} S_{11}(\underline{k}, \omega) / \operatorname{det} \underline{S}(\underline{k}, \omega),
$$

which has a pole where det $S=0$, corresponding to a collective mode. The zero sound nature of this pole is evident on taking the $k \rightarrow 0, \omega / k$ tinite limit of the Bose propagators. The zero sound velocity is found to be $v_{\mathrm{F}}^{*}\left(f_{0 / 3}^{\mathrm{S}}\right)^{1 / 2}$. More important for our purposes is a knowledge of the dispersion properties of 
this mode. This simplifies tremendously in the asymptotic limit of large mass enhancements, since the complicated momentum dependent terms in the Bose propagators in eq. (15) are suppressed by factors of $\left(1-n_{\mathrm{f}}\right)$. The dominant $k^{2}$ term arises from the backflow term in eq. (15) and the Bose propagators then take the form

$$
\begin{aligned}
& S_{11}(\underline{k}, \omega)=-\rho^{2}\left(2 t_{0}\right)^{2} J(\lambda)+Q k^{2} / 2 m, \\
& S_{12}(\underline{k}, \omega)=\rho\left[1-2 t_{0} J(\lambda)\right], \\
& S_{22}(\underline{k}, \omega)=-J(\lambda),
\end{aligned}
$$

for large mass enhancements. Here $J\left(\lambda=\omega / v_{\mathrm{F}}^{*} k\right)$ is the low momentum limit of the Lindhard function for the renormalized band. The collective mode then takes the following form for large mass enhancements,

$$
\omega=\left\{f_{0 / 3}^{\mathrm{S}}\right\}^{1 / 2} v_{\mathrm{F}}^{*} k+Q k^{2} / 2 m=v_{\mathrm{F}}^{*} k \lambda_{0}(k) .
$$

For finite $k$, the second term in eq. (22) dominates and the collective mode looks like a bare dispersion relation for the whole system of electrons. This behavior is unique to the Hubbard model. We do not expect this in the Anderson lattice where there is no backflow term. The corresponding term there represents the high energy fluctuations (the $\mathrm{f}^{1}$ peak in the spectrum). As for the particle-particle interaction, we find that the mediating propagator takes the form

$$
V(\omega, k)=\frac{\left[4 t_{0}-Q k^{2} / 2 m \rho^{2}\right]}{\left\{1-\left[4 t_{0}-\frac{Q k^{2}}{2 m \rho^{2}}\right] J(\lambda)\right\}},
$$

which near the zero sound pole takes the form

$$
V(k, \omega)=\frac{\lambda^{2}}{\left[\lambda^{2}-\lambda_{0}^{2}+\mathrm{i} \delta\right]}\left\{\frac{6 \lambda_{0}\left(k^{2}\right)}{N N^{*}(0)}\right\} .
$$

As mentioned earlier the $O(1 / N)$ exchange of a single density fluctuation does not produce a strong instability of any sort. Kotliar and Liu [22] found rather small superconducting coupling constants at this level. Our interest is in the $1 / N$ corrections to the Cooper instability and the spin exchange scattering amplitude - these are illustrated in fig. 4. The wavy lines represent Bose propagators and the straight lines represent Green's functions. The important feature is the internal frequency summation as this allows the zero sound component of the density fluctuation spectrum to contribute. Converting this frequency sum into an integration over energy we obtain

(a) a fermion pole part (from the poles in the Green functions) which picks out only the incoherent particle-hole part of the Bose propagator and is rather weak; and

(b) a contribution from the zero sound poles which results in the Cooper pair amplitude taking the following form

$$
\left\langle f_{m}^{+}\left(\underline{k}^{\prime}\right) \underline{f}_{m}^{+}\left(-\underline{k}^{\prime}\right) f_{m}(\underline{k}) f_{m}(-\underline{k})\right\rangle=I_{B}\left(\underline{k}, \underline{k}^{\prime}\right)
$$



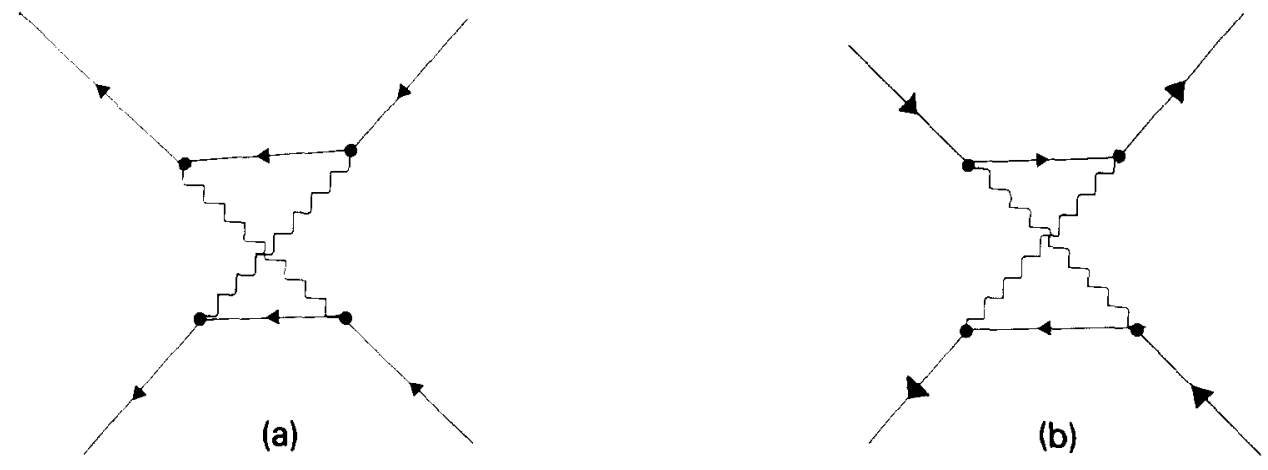

Fig. 4. $\mathrm{O}(1 / N)$ corrections to (a) the Cooper pair and (b) the spin exchange scattering amplitudes.

where

$$
I_{B}\left(k, k^{\prime}\right)=\frac{-3 Q}{8 N N^{*}(0) v_{\mathrm{F}}^{*} m \rho^{2}} \sum_{p} \frac{\lambda_{0}(p) p^{3}\left|p+k-k^{\prime}\right|^{2}}{\left(p^{4}-\left|p+k-k^{\prime}\right|^{4}\right)}+\left(k \leftrightarrow k^{\prime}\right),
$$

while the spin exchange scattering amplitude is simply

$$
\left\langle f_{m}^{+}\left(k_{2}-k\right) f_{m}^{+}\left(k_{1}+k\right) f_{m_{2}}\left(k_{2}\right) f_{m_{1}}\left(k_{1}\right)\right\rangle_{k \rightarrow 0}=-I_{B}\left(k_{1}, k_{2}\right) \text {. }
$$

For the Cooper amplitude, we can evaluate the integral directly for equal momenta and obtain

$$
N^{*}(0) I_{B}\left(k_{1}, k_{1}\right)=-\frac{18 Q^{3}}{320 N^{2}}\left(\frac{m^{*}}{m}\right)^{3}
$$

which is repulsive and strong, varying as $\left(m^{*} / m\right)^{3}$. Such a strong repulsion has been found in another theory involving two loop exchange, namely two plasmon exchange in the electron gas. Grabowski and Sham [23] found that such processes won out over the one loop plasmon exchange, which by itself favored superconductivity.

In conclusion, we have examined in this paper two opposite viewpoints regarding actinide and rare earth systems. The single impurity Anderson model with two magnetic configurations has been treated along lines extending earlicr $1 / N$ expansion work to next leading order in a manner that respects the restrictions imposed by Ward identities and scale invariance. The $1 / N$ expansion has also been carried out to second order for a lattice model of relevance to actinide and high- $T_{\mathrm{c}}$ systems, namely the infinite $U$ Hubbard model. We find in three dimensions a strong ferromagnetic contribution to the scattering amplitudes arising from virtual exchange of two collective modes, near half filling. There seems no reason not to expect the same process to be important in two dimensions for a square lattice, but this remains to be carried out.

\section{References}

[1] P.W. Anderson, in: Proc. Enrico Fermi Int. School of Physics, Frontiers and Borderlines in Many Particle Physics, Varenna (North-Holland, 1987).

[2] D.M. Newns, M. Rasolt, P. Pattnaik and D. Papaconstantopoulos, Phys. Rev. B 38 (1989) 7033.

[3] P.W. Anderson, Phys. Rev. Lett. 65 (1990) 2306;

M. Ogata and H. Shiba, Phys. Rev. B 41 (1990) 2326. 
[4] N.E. Bickers, Rev. Mod. Phys. 59 (1987) 845.

[5] A. Yoshimori, Prog. Theor. Phys. 55 (1976) 67; P. Schlottmann, Phys. Lett. A 100 (1984) 509.

[6] J.H. Jefferson, J. Phys. C 10 (1977) 3589; F.D.M. Haldane, Phys. Rev. Lett. 40 (1978) 416.

[7] B. Doucot and R. Rammal, Int. J. Mod. Phys. B 3 (1989) 1755;

B.S. Shastry, H.R. Krishnamurthy and P.W. Anderson, Phys. Rev. B 41 (1990) 2375;

A. Barbieri, J. Riera and A.P. Young, Phys. Rev. B 41 (1990).

[8] Y. Nagaoka, Phys. Rev. 147 (1966) 392.

[9] A. Auerbach and K. Levin, Phys. Rev. Lett. 57 (1986) 877;

A. Millis and P. A. Lee, Phys. Rev. B 35 (1986) 3394

[10] A. Houghton, N. Read and H. Won, Phys. Rev. B 37 (1988) 3782.

[11] A. Mazzafero, C.A. Balseiro and B.A. Alascio, Phys. Rev. Lett. 47 (1981) 274.

[12] P. Schlottmann, Phys. Rep. 181 (1989) 1.

[13] D.L. Cox, Phys. Rev. Lett. 59 (1987) 1240.

[14] Y. Yafet, C.M. Varma and B. Jones, Phys. Rev. B 32 (1985) 360.

[15] N. Read, K. Dharmvir, J.W. Rasul and D.M. Newns, J. Phys. C 19 (1986) 15097.

[16] A.C. Nunes, J.W. Rasul and G.A. Gehring, J. Phys. C 19 (1986) 1017.

[17] S.M.M. Evans and G. A. Gehring, J. Phys. C 1 (1989) 3095.

[18] T. Saso, J. Magn. \& Magn. Mater. 76-77 (1988) 176.

[19] Y. Shimizu, O. Sakai and T. Kasuya, Physica C 163 (1990) 401.

[20] H. Keiter and J.C. Kimball, Int. J. Magn. 1 (1970) 233.

[21] T.V. Ramakrishnan, J. Phys. C 19 (1986) L1;

N. Read and D.M. Newns, Adv. Phys. 36 (1987) 799

[22] G. Kotliar and J. Liu, Phys. Rev. Lett. 61 (1988) 1784.

[23] M. Grabowski and L.J. Sham, Rev. B 29 (1984) 6132. 